\title{
An Analytical Study on Cerebral State Induced by Changes in Auditory Stimulation
}

\author{
Jeong-Hoon Shin \\ School of Information Technology, Catholic University of Daegu \\ only4you@cu.ac.kr
}

\begin{abstract}
The sense of hearing begins in the fetus, and the auditory organs of a newborn baby are able to perceive sounds from its surrounding environment immediately after birth. Due to the characteristics of these auditory organs, sound plays an important role in language learning and brain development of children who are exposed to various stimuli, such as the sound of their mother's voice, nature, and their immediate environment. During this stage of development, the human brain is in the process of learning. Auditory stimuli which at this young age are combined systematically with experience and integrated into memory. Thus, various auditory stimuli which human beings are exposed to in their daily lives induce unconscious responses from the cerebral learning area which they do not consciously remember, which leads to changes in the cerebral activation state.[1],[2] The purpose of this study was to investigate the effect of auditory stimuli of a specific frequency on the human cerebrum, by examining the activation state in subjects after presenting them with auditory stimuli across audible frequency bands, and to analyze the results. The results of this study on the cerebral activation state, in response to auditory stimulation will provide basic data for expanding applications to various fields, such as neuro-feedback, which taps into auditory stimulation for the treatment of mental illnesses.
\end{abstract}

Keywords: brain wave, neuro-feedback, auditory stimulus, cerebral activation

\section{Introduction}

Brain waves are electrical signals within the brain that are generated by neurons. As brain waves are affected by the activity of nerve cells [1], brain waves may represent brain activities. With technology for brain wave measurement being taken to the next level, the sectors which involve the analysis, control, and application of brain waves have been expanding. Typical examples of that may include applications in the medical, educational, and training fields. Particularly, the health care field has seen continuous efforts in connection with the prevention and treatment of mental illnesses in patients through the application of the results of brain wave analysis and control of the cerebral activation state [3].

Treatment and prevention of mental illnesses based on brain wave data have various advantages, compared to conventional surgery or pharmacological protocols, and as a result, are expected to find wider applications in the period ahead. The most typical advantage, brought by analysis and control of brain waves in the treatment and prevention of mental illnesses, is that patients can avoid side effects from existing surgery or medication. Surgery and drug administration methods, the main treatment methods in contemporary medicine, are likely to give rise to side effects due to a patient's condition, or via continuous medication. Those side effects are even more problematic because they are not uniform in all patients, and are not easy to predict in terms of symptoms,

Received (July 15, 2017), Review Result (October 20, 2017), Accepted (October 24, 2017) 
depending on type of surgery, type of drugs, duration of administration, etc. For those reasons, people in contemporary society prefer non-surgical/non-pharmacological treatment methods which incur little risk of side effects, rather than surgical/ pharmacological treatments with potential side effects, and the most typical field is the neuro-feedback clinical treatment. Neuro-feedback therapy is a method by which the cerebral activation state of a patient is analyzed and compared with that of a normal person, and based on the results, therapists train patients on how to control and maintain their cerebral activation state. However, most patients may give up after training because it is not easy for them to learn how to control their cerebral activation. Therefore, improvements need to be made.

This paper has presented a method for controlling the cerebral activation state of patients by using audiovisual stimulation, which is also a method used in the field of neuro- feedback therapy and training, to help overcome disadvantages of existing systems. For that, the cerebral activation status of subjects needs to be analyzed by using various auditory stimuli. If the auditory stimulation control technique used in this study and results of the analysis are applied, the problems of existing neuro-feedback therapy techniques may be resolved, and more proper treatment and training will be able to be provided to patients.

\section{Related Studies}

\subsection{Combined Mapping of Human Auditory EEG and MEG Responses [1]}

In this paper, auditory electric and magnetic P50(m), N1(m) and $\mathrm{MMN}(\mathrm{m})$ responses to standard, deviant and novel sounds were studied by recording brain electrical activity with 25 EEG electrodes simultaneously with the corresponding magnetic signals measured with 122 MEG gradiometer coils. The sources of these responses were located on the basis of the MEG responses; all were found to be in the supra-temporal plane. The goal of this paper was to investigate to what degree the source locations and orientations determined from the magnetic data account for the measured EEG signals. It was found that the electric P50, N1 and MMN responses can to a considerable degree be explained by the sources of the corresponding magnetic responses. In addition, source-current components not detectable by MEG were shown to contribute to the measured EEG signals.

\subsection{Auditory Evoked Potential Response and Hearing Loss: A Review [2]}

This paper says that Hypoacusis is the most prevalent sensory disability in the world and consequently, it can lead to impede speech in human beings. One best approach to tackle this issue is to conduct early and effective hearing screening test using Electroencephalogram (EEG). EEG based hearing threshold level determination is most suitable for persons who lack verbal communication and behavioral response to sound stimulation. Auditory evoked potential (AEP) is a type of EEG signal emanated from the brain scalp by an acoustical stimulus. The goal of this review is to assess the current state of knowledge in estimating the hearing threshold levels based on AEP response. AEP response reflects the auditory ability level of an individual. An intelligent hearing perception level system enables to examine and determine the functional integrity of the auditory system. Systematic evaluation of EEG based hearing perception level system predicting the hearing loss in newborns, infants and multiple handicaps will be a priority of interest for future research. 


\section{Methods}

\subsection{Selection of Stimulus Sound Sources in Consideration of Audible Frequency Characteristics}

Human auditory organs can hear sounds ranging from $20 \mathrm{~Hz}$ to $20 \mathrm{KHz}$ bandwidth, but the sensitivity of the auditory sense is not uniform for all frequencies. Each frequency in the audible frequency bandwidth has a minimum threshold, and the sound source in each bandwidth can be perceived by sensory organs when the stimulus is applied at an intensity above the minimum threshold. For example, when a person is exposed to "Sound A" in the $1000 \mathrm{~Hz}$ bandwidth, and "Sound B" in the $20000 \mathrm{~Hz}$ bandwidth, the minimum stimulus intensity that said person can perceive is different. For both sounds to be perceived by humans, the B-stimulus source needs to stimulate the auditory organ at a higher intensity. [3] [5] In general, sensitivity of the human auditory organ is low in the low frequency range and increases as the frequency rises. Sensitivity is best near $3 \mathrm{kHz}$ and decreases in frequency ranges above that. Usually, a unit called a 'Loudness Level' is used to express such characteristics. This represents the sound pressure level value of pure tone in the $1000 \mathrm{~Hz}$ band, which a person with normal auditory acuity thinks has the same volume as that of the sound. The difference between auditory sensitivity to low frequency bandwidths, and auditory sensitivity to high frequency bandwidths becomes smaller as the level of sound increases. Low frequency sounds are not very audible when we listen to music. However, the sound in low frequency will be clearly audible if we increase the volume. This phenomenon is attributed to the correlation between audible frequency characteristics and sound pressure. Figure 1 and Figure 2 below present the equal loudness contours and human hearing threshold curves, which show the sound pressure intensity characteristics variable, depending on human auditory stimulation frequencies[6] [8].

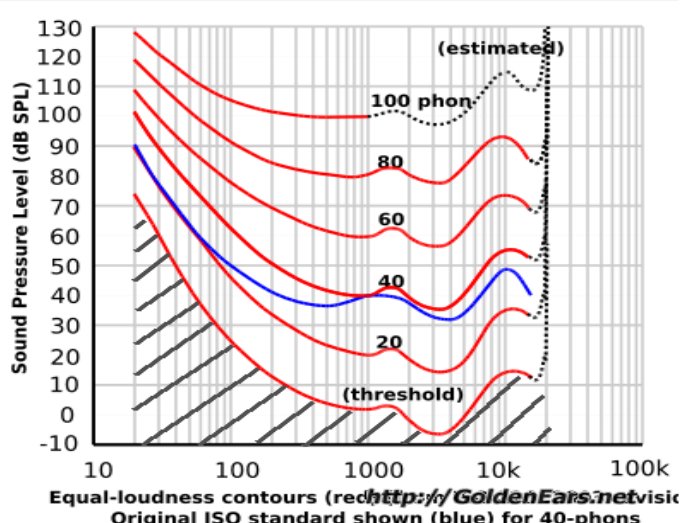

Figure 1. Equal Loudness Contours

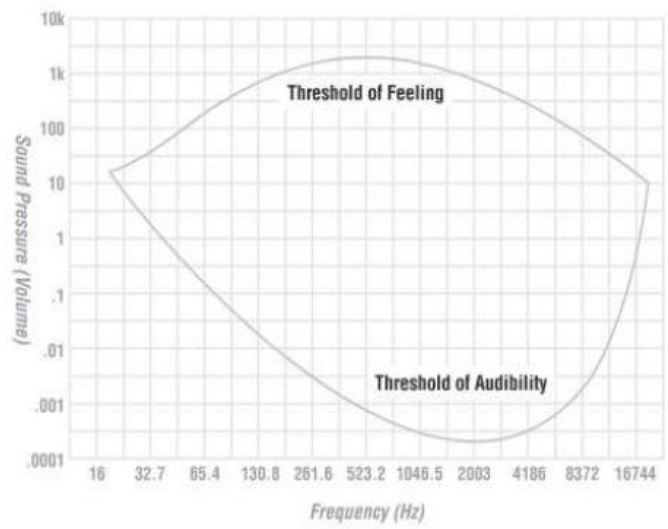

Figure 2. Human Hearing Threshold Curve

In this study, we have divided the auditory stimulation frequencies into 25 critical bandwidths, considering the discrimination limit of the human auditory organ, relative to frequency, sound intensity, detection of sound and amplitude modulation, and the limit of frequency characteristic variations, etc. As shown in Table [1], center frequencies of 25 critical bandwidths were adopted to present substantial stimulus sound sources to the subjects. 
Table 1. Critical Bandwidth and Center Frequency

\begin{tabular}{|c|c|c|c|c|c|c|c|}
\hline $\begin{array}{l}\text { Critical } \\
\text { Band }(\mathbf{H z})\end{array}$ & $\begin{array}{l}\text { Center } \\
\text { frequency }\end{array}$ & $\begin{array}{l}\text { Critical } \\
\text { Band }(\mathbf{H z})\end{array}$ & $\begin{array}{l}\text { Center } \\
\text { frequency }\end{array}$ & $\begin{array}{l}\text { Critical } \\
\text { Band(Hz) }\end{array}$ & $\begin{array}{l}\text { Center } \\
\text { frequency }\end{array}$ & $\begin{array}{l}\text { Critical } \\
\text { Band(Hz) }\end{array}$ & $\begin{array}{l}\text { Center } \\
\text { frequency }\end{array}$ \\
\hline $0 \sim 100$ & $50 \mathrm{hz}$ & $100 \sim 200$ & $150 \mathrm{hz}$ & $200 \sim 300$ & $250 \mathrm{hz}$ & $300 \sim 400$ & $350 \mathrm{hz}$ \\
\hline $400 \sim 510$ & $455 \mathrm{hz}$ & $510 \sim 630$ & $570 \mathrm{hz}$ & $630 \sim 770$ & $700 \mathrm{hz}$ & $770 \sim 920$ & $845 \mathrm{hz}$ \\
\hline $920 \sim 1080$ & $1,000 \mathrm{hz}$ & $1080 \sim 1270$ & $1,175 \mathrm{hz}$ & $1270 \sim 1480$ & $1,375 \mathrm{hz}$ & $1480 \sim 1720$ & $1,600 \mathrm{hz}$ \\
\hline $1720 \sim 2000$ & $1,860 \mathrm{hz}$ & $2000 \sim 2320$ & $2,160 \mathrm{hz}$ & $2320 \sim 2700$ & $2,510 \mathrm{hz}$ & $2700 \sim 3150$ & $2,925 \mathrm{hz}$ \\
\hline $3150 \sim 3700$ & $3,425 \mathrm{hz}$ & $3700 \sim 4400$ & $4,050 \mathrm{hz}$ & $4400 \sim 5300$ & $4,850 \mathrm{hz}$ & $5300 \sim 6400$ & $5,850 \mathrm{hz}$ \\
\hline $6400 \sim 7700$ & $7,050 \mathrm{hz}$ & $7700 \sim 9500$ & $8,600 \mathrm{hz}$ & $9500 \sim 12000$ & $10,750 \mathrm{hz}$ & $12000 \sim 15500$ & $13,750 \mathrm{hz}$ \\
\hline $15500 \sim 22500$ & $19,000 \mathrm{hz}$ & & & & & & \\
\hline
\end{tabular}

\subsection{Subjects, Stimulation, and Recording}

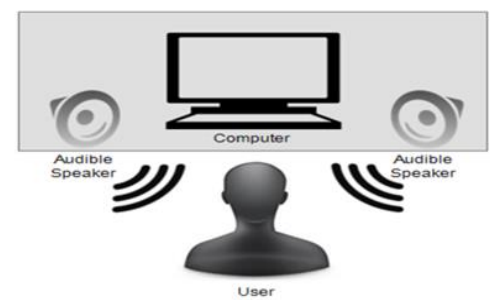

Figure 3. Experiment Environment
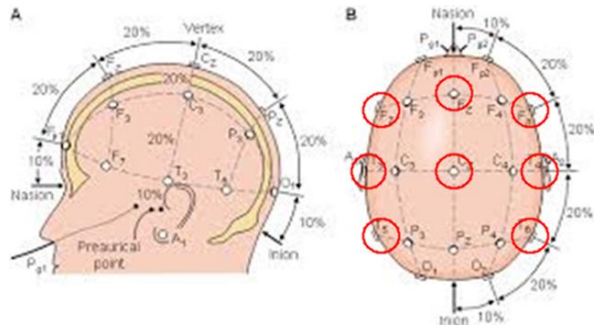

Figure 4. Electrode Placement Diagram

In this study, we recruited 60 healthy individuals, consisting of 30 men and 30 women in their 20 s to 30 s, who were capable of sensing sounds within a wide audible frequency range and having no abnormalities in their auditory organs, to minimize the possibility of damage to auditory organs during experimentation.

An independent space, which took the shape illustrated in Fig. 3, was configured to ensure stable brain wave signal data collection. We collected brain wave signals reflecting the cerebral activation, depending on the brain wave signals and auditory stimulation in the stable section, while the influence of external noise was minimized. For electrode arrangement necessary to collect brain wave data from subjects, 10 points were used, including the reference electrode $\mathrm{A} 1$ and $\mathrm{A} 2$, and 8 points (F7, F8, T3, T4, T5, T6, Fz, and $\mathrm{Cz}$ ) which could reflect not only the effects on auditory organs, but also the characteristics of the frontal, temporal, and occipital lobes. Moreover, subjects were stimulated with center frequencies using critical bandwidths, and at the same time, the subjects' brain wave signals were collected every 10 seconds at a sample rate of $256 \mathrm{~Hz}$. In addition, LPF with a $50 \mathrm{~Hz}$ cut-off frequency was used to remove noise other than brain wave signals.

\subsection{Data Analysis}

Quantitative analysis was performed on collected brain wave signals by channels to examine the effect of each stimulus source on the activation state of the whole brain.

6 frequency bandwidths were used, including delta waves, theta waves, alpha waves, beta waves, gamma waves, and SMR waves, which have commonly been used in brain wave signal analysis, and neuro-feedback therapy/training fields. For the collected brain wave signals, we analyzed the relative energy value for each frequency bandwidth by performing FFT calculation with a 1 second interval. Additionally, we analyzed average relative energy for 10 seconds by repeatedly performing calculations when the same sound source was provided. After analyzing the average relative energy by bandwidth for 10 seconds for 25 sound sources, we analyzed each subject. During this analysis, we 
attempted to determine which sound would represent the maximum/minimum average relative energy of the 6 frequency bandwidths for each subject. After repeating this process for 60 subjects, the results of the statistical analysis were recorded.

\section{Results}

The main purpose of this study was to verify whether any uniform characteristics would exist, depending on different sounds, in the event that any change in human cerebral activation state was caused by auditory stimuli, and to analyze the patterns of any possible changes, and the experiment was conducted accordingly. That aimed to broaden applications to various fields requiring control of cerebral activation state by utilizing external stimuli, such as mental illness treatment and prevention, with audiovisual stimulation and neuro-feedback therapy training, for example. In this study, the reproducibility of cerebral activation status by region was analyzed, depending on sound source, to achieve the aforesaid purpose. Figures 5 through 12 below presents the identical response pattern of the cerebral activation state in users, depending on the sound source, as cumulative numbers. Yellow indicates that an identical response pattern registered at $15 \%$ or higher among 60 total subjects.

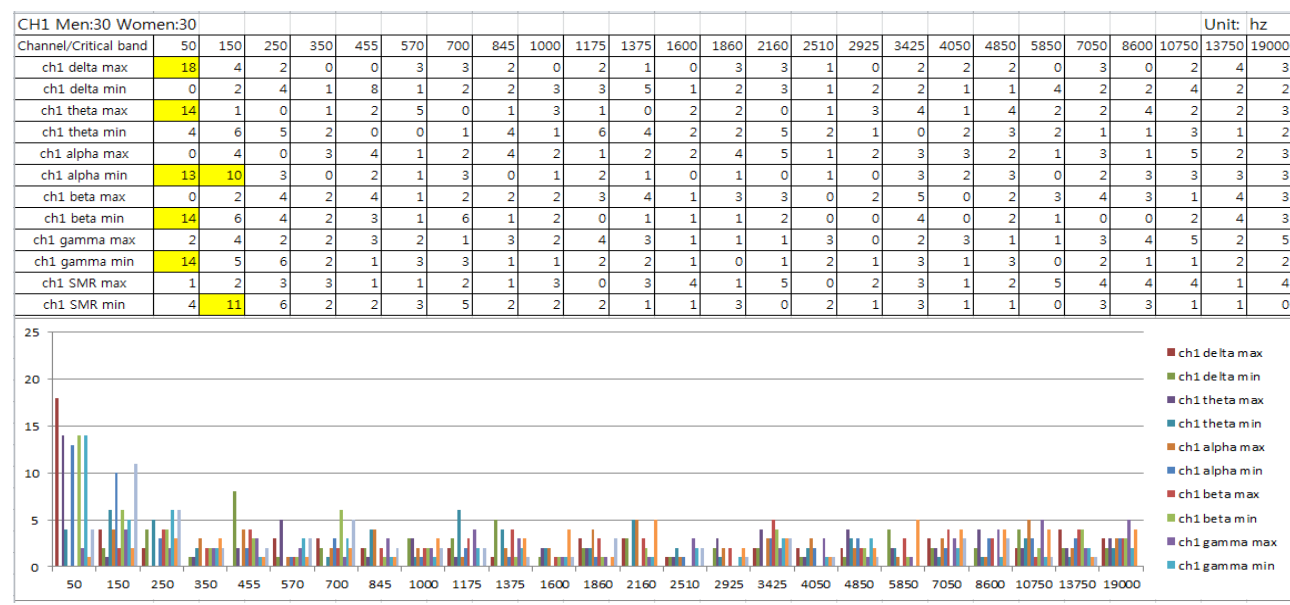

Figure 5. Statistical Reproducibility (60 men and women in total/ Channel 1 / F7)

The pattern of changes in the cerebral activation state in various regions was presented in Figure 5 based on the International Electrode Placement Method. Figure 5 shows that only 2 sound sources, at $50 \mathrm{~Hz}$ and $150 \mathrm{~Hz}$ respectively, exhibited a slightly higher reproducibility among the 25 sound sources. At $50 \mathrm{~Hz}$, the relative energy values of the delta and theta bands in the F7 region rose to the highest level among the 25 sounds. In addition, the $50 \mathrm{~Hz}$ test showed a response that minimized the relative energy values of alpha, beta, and gamma bandwidths in the F7 region, from among the 25 sound sources. Moreover, the $150 \mathrm{~Hz}$ test showed a response that minimized the relative energy values of alpha and SMR wavelengths in the F7 region among the 25 sound sources. 


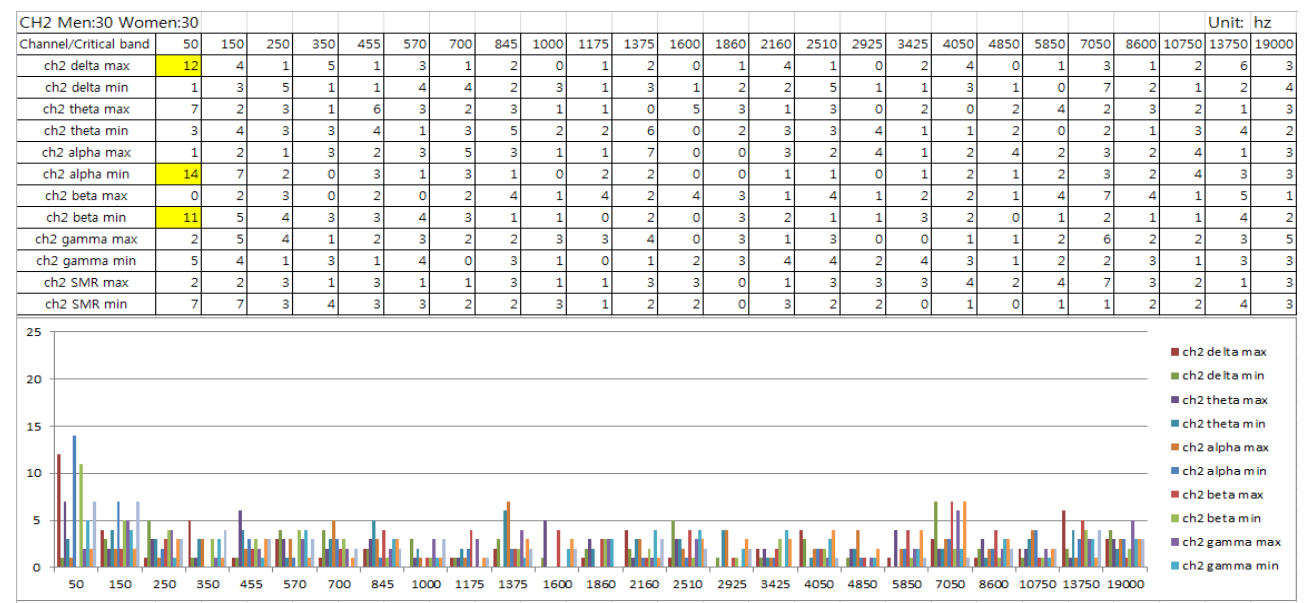

Figure 6. Statistical Reproducibility (60 men and women in total/ Channel 2 / F8)

The pattern of changes in the cerebral activation state in the F8 region is presented in Figure 6. In the F8 region, the relative energy of the delta wavelength showed a maximum response for the $50 \mathrm{~Hz}$ stimulus sound source while the relative energy of the alpha wave and beta wavelengths showed a minimum response. Besides, it could be found that no significant statistical reproducibility may be manifested for the 24 stimulus sound sources other than using $50 \mathrm{~Hz}$.

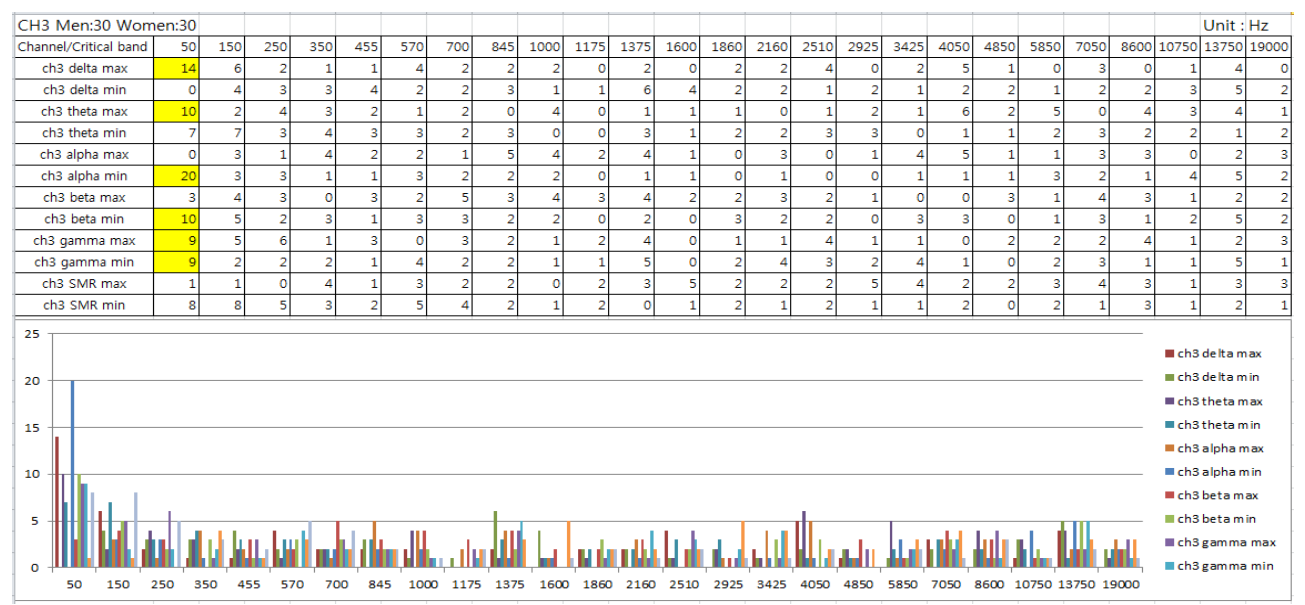

Figure 7. Statistical Reproducibility (60 men and women in total/ Channel 3 / T3)

We analyzed the patterns of changes in the cerebral activation state variables depending on stimulus sound sources in the T3 region, illustrated in Figure 7. The results showed that $50 \mathrm{~Hz}$ stimuli made the relative energy value in the delta and theta wave bandwidths showed the maximum value. The relative energy values in the alpha and beta wave bandwidths were minimal. The results of the analysis showed that the relative energy value in the gamma wave bandwidth tended to fluctuate to the maximum and minimum simultaneously. That would necessitate analysis on factors of change in additional cerebral activation states, as a polarization phenomenon arising from subjects/characteristics.

We analyzed the pattern of changes in the cerebral activation state variables depending on stimulus sound sources in the T4 region, illustrated in Figure 8. The results showed that $50 \mathrm{~Hz}$ stimulus sound source maximized the relative energy value for the delta and theta wave bandwidths, and minimized the relative energy value in the alpha, beta, and 
SMR wavelengths. Furthermore, it was found that the $150 \mathrm{~Hz}$ stimulus sound source additionally minimized the relative energy value of the SMR wave band.

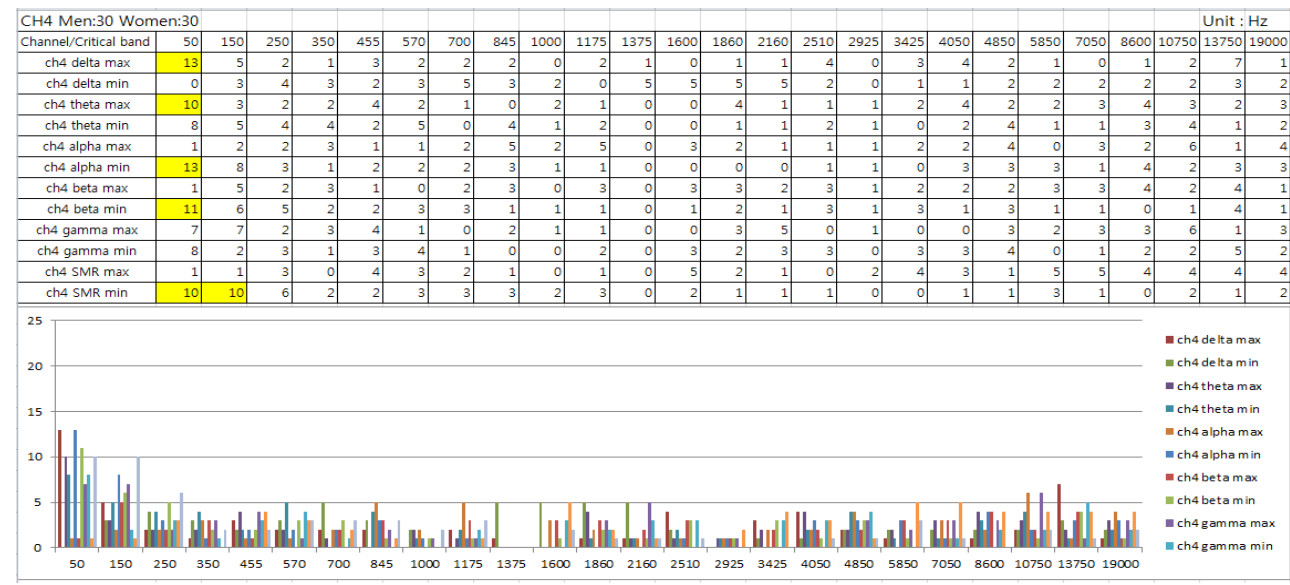

Figure 8. Statistical Reproducibility (60 men and women in total/ Channel 4 / T4)

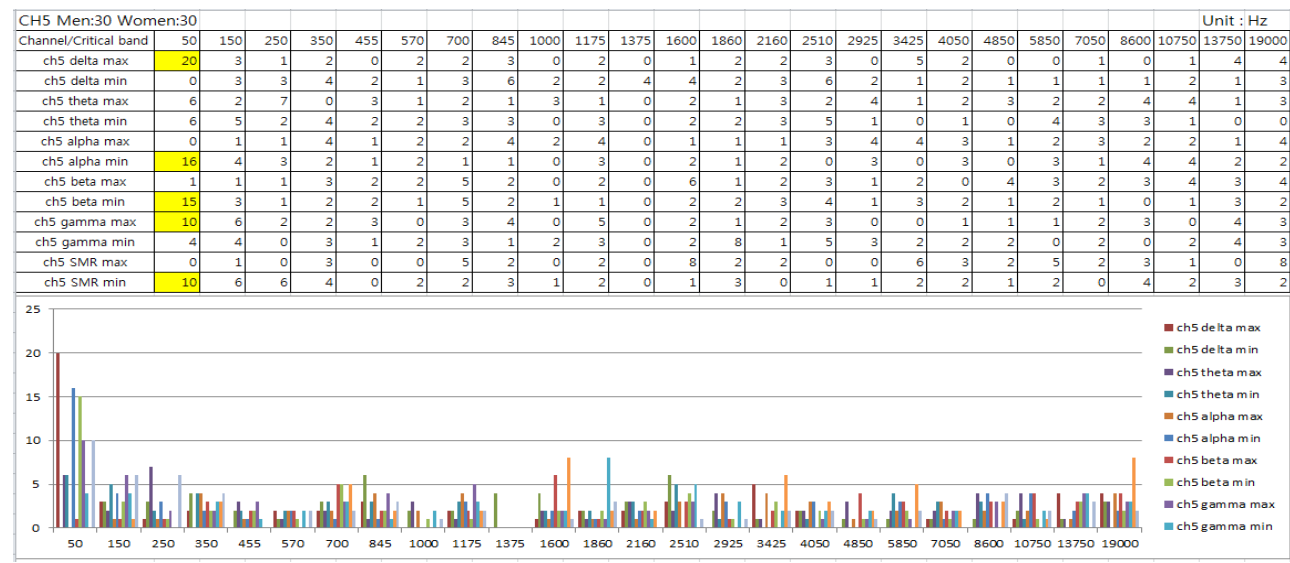

Figure 9. Statistical Reproducibility (60 men and women in total/ Channel 5 / T5)

Figure 9 which presents the pattern of changes in the T5 region, it was shown that the $50 \mathrm{~Hz}$ stimulus sound source maximized the relative energy of delta and gamma wavelengths, and minimized the relative energy of alpha, beta, and SMR wavelengths.

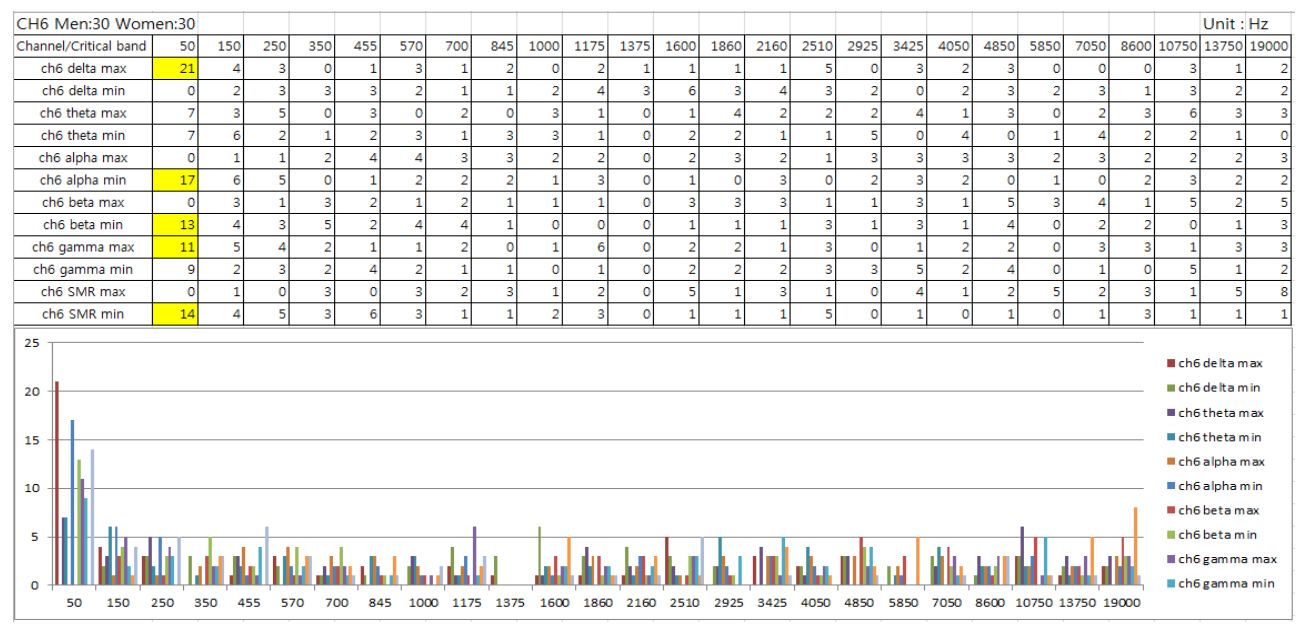

Figure 10. Statistical Reproducibility (60 men and women in total/ Channel 6 / T6) 
Based on the results of the analysis on the cerebral activation state in the T6 region, illustrated in Figure 10,5 the $0 \mathrm{~Hz}$ stimulus sound source maximized the relative energy values in delta and gamma wavelengths. The relative energy values were minimized in alpha, beta, and SMR wavelengths.

Based on Figure 11, we determined the effect of the $50 \mathrm{~Hz}$ stimulus sound source on the changes in cerebral activation state in the Fz region. In other words, at $50 \mathrm{~Hz}$, the sound maximized the relative energy value of delta wavelength, and minimized the relative energy value of the alpha, beta, and SMR wavelengths.

Considering the pattern of changes in the cerebral activation state in the $\mathrm{Cz}$ region, as shown in Figure 12, it was noted that the stimulus sound source of the $50 \mathrm{~Hz}$ bandwidth maximized the relative energy value of the delta wave band and minimized the relative energy value of the alpha and beta wavelengths.

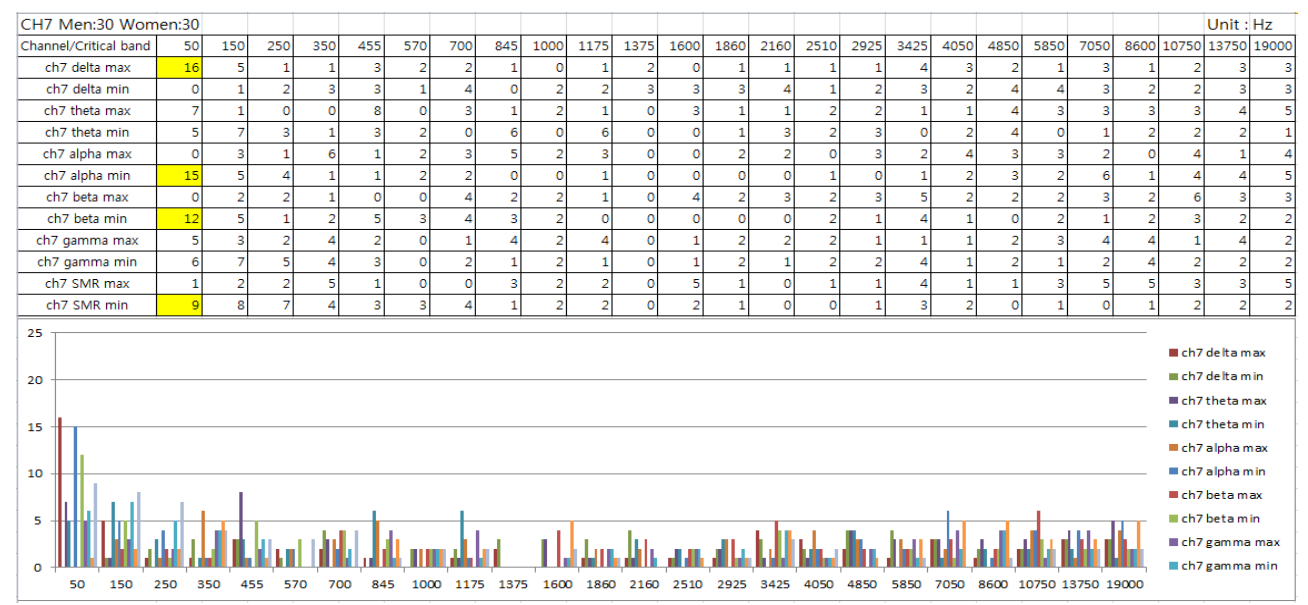

Figure 11. Statistical Reproducibility (60 men and women in total/ Channel 7 / Fz)

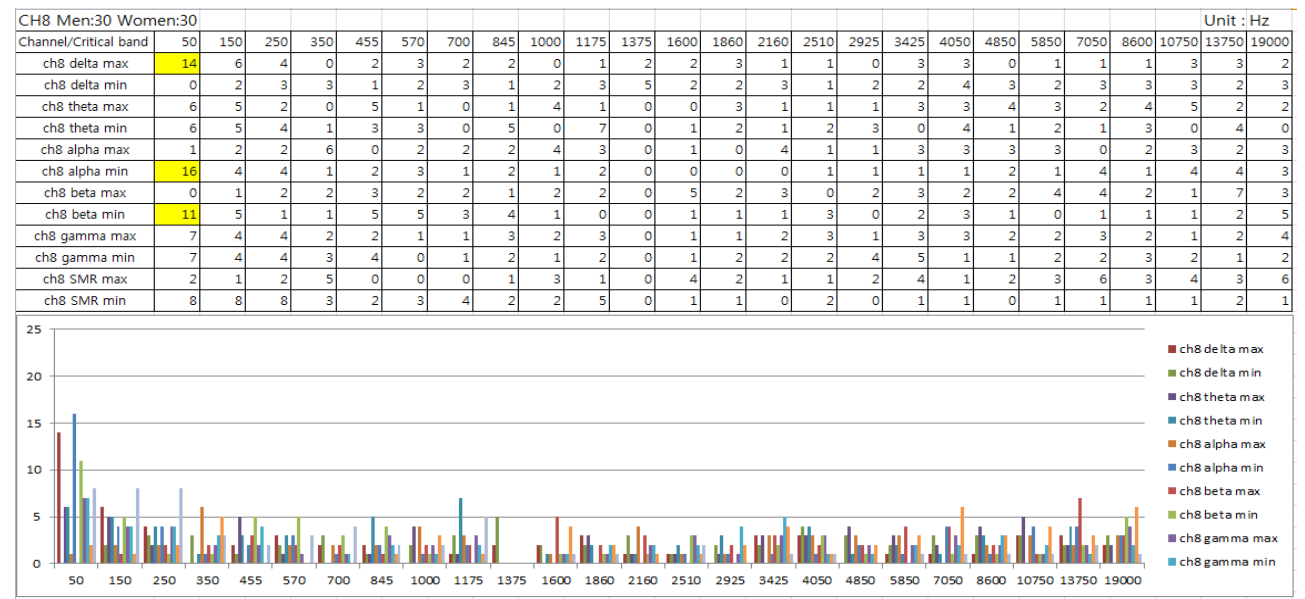

Figure 12. Statistical Reproducibility (60 men and women in total/ Channel 8 / Cz)

\section{Conclusions}

The results of this study indicated that auditory stimuli within specific frequency bandwidths applied to human auditory organs led to common changes in the cerebral activation state of many subjects. These sounds, which fell within the scope of most audible frequency bandwidths, induced different responses in cerebral activation states among subjects, thus failing to show statistically significant reproducibility.

Table 2 summarizes the pattern of changes in cerebral activation state based on stimulation from 2 different types of sound sources $(50 \mathrm{~Hz}$ and $150 \mathrm{~Hz})$, which were 
found to exhibit statistically significant reproducibility among the stimulus sounds used in this study.

As shown in Table 2, the $50 \mathrm{~Hz}$ frequency bandwidth exhibited noteworthy changes in the activation state across the cerebral region, while the $150 \mathrm{~Hz}$ frequency region exhibited significant reproducibility in some cerebral regions. This was attributable to the fact that the characteristic factors related to the sensory organs of the subjects acted as filters for the characteristics of the stimulus sound sources, thus resulting in additional influence on the cerebral learning state of things subjects had previously learned, while the auditory stimulus sounds were transmitted to the brain, and affected the cerebral activation state. In other words, if a special experience has been learned and integrated into the memory of subjects, along with auditory stimulation of specific frequency bandwidths in their daily lives, the auditory stimulus provided to the subjects would induce the memory of learned experiences to affect the changes in the cerebral activation state, besides the influence of the auditory stimulus itself. As stimulus sound sources corresponding to $150 \mathrm{~Hz}$ are more likely to have already been learned in daily lives, compared to sounds in the very low $50 \mathrm{~Hz}$ frequency range, sounds in the $150 \mathrm{~Hz}$ range are considered to be unable to exhibit statistically significant reproducibility. This logic also constitutes the reason why the stimulus sound sources other than $50 \mathrm{~Hz}$ and $150 \mathrm{~Hz}$ frequency bandwidths have no significant reproducibility among the 25 stimulus sound sources in the audible frequency bandwidth. The components of daily noises that subjects experience in everyday life consist of the sounds corresponding to frequencies equal to or exceeding $250 \mathrm{~Hz}$, rather than sounds of very low frequencies such as $50 \mathrm{~Hz}$ and $150 \mathrm{~Hz}$. That is attributable to the high likelihood that those sounds have already been learned by the brain of the subjects.

If specific stimulus sound sources have already been thus integrated by the brains of the subjects through their everyday lives, additional factors of change, which vary from person to person, will be manifested, i.e., the changes in characteristics of the cerebral activation state, which reflect the cerebral learning state, besides the changes in characteristics which are induced by stimulus sound sources, in the process by which specific auditory stimuli affect the changes in the cerebral activation state of subjects.

Table 2. Changes in Cerebral Activation State, Induced by Stimulus Sound Sources, for each Cerebral Region

$50 \mathrm{~Hz}$ stimulus sound source, $150 \mathrm{~Hz}$ stimulus sound source

\begin{tabular}{|l|l|l|l|}
\hline & \multicolumn{1}{|c|}{$\begin{array}{c}\text { Left Cerebral } \\
\text { Hemisphere }\end{array}$} & Cerebral Center & \multicolumn{1}{c|}{$\begin{array}{c}\text { Right Cerebral } \\
\text { Hemisphere }\end{array}$} \\
\hline $\begin{array}{l}\text { Anterior } \\
\text { temporal }\end{array}$ & $\begin{array}{l}\text { delta max } \\
\text { theta max } \\
\text { alpha min alpha min } \\
\text { beta min } \\
\text { gamma min } \\
\text { SMR min }\end{array}$ & $\begin{array}{l}\text { delta max } \\
\text { alpha min } \\
\text { beta min }\end{array}$ & $\begin{array}{l}\text { delta max } \\
\text { alpha min } \\
\text { beta min }\end{array}$ \\
\hline $\begin{array}{l}\text { Middle } \\
\text { temporal }\end{array}$ & $\begin{array}{l}\text { delta max } \\
\text { theta max } \\
\text { alpha min } \\
\text { beta min } \\
\text { gamma max \& min }\end{array}$ & SMR min & delta max \\
Posterior \\
temporal
\end{tabular}




\section{Acknowledgments}

This work was supported by research grants from the Catholic University of Daegu.

\section{References}

[1] Istva'n Winklera.b, Kimmo Alhoa.c, Carles Escerac and Minna Huotilainena. "Combined mapping of human auditory EEG and MEG responses”, Electroencephalography and clinical Neurophysiology, vol. 108, (1998), pp. 370-379.

[2] J. F. Lubar, M. O. Swartwood, J. N. Swartwood and D. L. Timmermann, "Quantitative EEG and Auditory Event-Related Potentials in the Evaluation of Attention-Deficit/Hyperactivity Disorder: Effects of Methylphenidate and Implications for Neurofeedback Training", Journal of Psychoeducational Assessment, ADHD Special, (1995), pp. 143-160.

[3] M. P. Paulraj, K. Subramaniam, S. Bin Yaccob, A. H. Bin Adom and C. R. Hema, "Auditory Evoked Potential Response and Hearing Loss: A Review", The Open Biomedical Engineering Journal, vol. 9, (2015), pp. 17-24.

[4] T. Harmony, G. Hinojosa, E. Marosi, J. Becker, M. Rodriguez, A. Reyes and C. Rocha, "Correlation between EEG spectral parameters and an educational evaluation”, International Journal of Neuroscience, vol. 54, (1990), pp. 147-155.

[5] C. Mulert, L. Ja"ger, S. Propp, S. Karch, S. Sto“rmann, O. Pogarell, H.-J. Mo“ ller, G. Juckel and Ulrich Hegerla, "Sound level dependence of the primary auditory cortex: Simultaneous measurement with 61channel EEG and fMRI", NeuroImage, vol. 28, (2005), pp. 49-58.

[6] N. Ille, P. Berg and M. Scherg, "Artifact correction of the ongoing EEG using spatial filters based on artifact and brain signal topographies", J. Clin. Neurophysiol, vol. 19, (2002), pp. 113-124.

[7] X. Kang, O. Bertrand, K. Alho, E. W. Yund, T. J. Herron and D. L. Woods, "Local landmark-based mapping of human auditory cortex", NeuroImage, vol. 22, (2004), pp. 1657-1670.

[8] V. L. Towle, J. Bolanos, D. Suarez, K. Tan, R. Grzeszczuk, D. N. Levin, R. Cakmur, S. A. Frank and J. P. Spire, "Location of EEG electrodes: locating the best-fitting sphere relative to cortical anatomy", Electroencephalogr. Clin. Neurophysiol., vol. 86, (1993), pp. 1-6. 